\title{
Image matching algorithms for breech face marks and firing pins in a database of spent cartridge cases of firearms
}

\author{
Zeno Geradts $^{\mathrm{a}}$, Jurrien Bijhold ${ }^{\mathrm{a}}$, Rob Hermsen ${ }^{\mathrm{a}}$, Fionn Murtagh ${ }^{\mathrm{b}}$ \\ ${ }^{a}$ Netherlands Forensic Institute of the Ministry of Justice* \\ Volmerlaan 17, 2288 GD Rijswijk, Netherlands \\ ${ }^{\mathrm{b}}$ Queen's University of Belfast \\ BT7 1NN Belfast \\ Northern Ireland
}

\begin{abstract}
On the market several systems exist for collecting spent ammunition data for forensic investigation. These databases store images of cartridge cases and the marks on them. Image matching is used to create hit lists that show those cartridges in the database which have marks that are most similar to the marks of the cartridge case under investigation. The research in this paper focuses on the different methods of feature selection and pattern recognition that can be used for optimizing the results of image matching. A fast pre-selection method based on signatures is applied that is based on the Kanade Lucas Tomasi (KLT) equation. The positions of the points computed with this method are compared. In this way 11 of the 49 images were in the top position in combination with the third scale of the à trous wavelet. Light conditions and the prominence of the marks determines to a large extent whether correct matches are found in the top ranked position. All images were retrieved in the top five percent of the complete database. This method takes only a few minutes, which can be structured for comparisons to be carried out in seconds.
\end{abstract}

Keywords: Forensic Science, firearms, image matching, image database, cartridge case, wavelets, pattern recognition

\section{INTRODUCTION}

In the Netherlands Forensic Institute (NFI) a research study has been carried out for automated comparison algorithms of cartridge cases. This study is a part of an evaluation of the different systems on the market for handling databases of cartridge cases and bullets.

The reason to compare the different methods of image matching is that the methods are proprietary. For use in a forensic laboratory it is important for quality assurance to understand why a certain image is not found in top matching ranks and to have more background on the image-matching engine. Another reason for this research is to improve the results of image matching.

\section{FORENSIC EXAMINATION}

When a firearm is loaded and fired the mechanisms and part in the firearm that come into contact with the cartridge case cause impressions and striations that can be characteristic for the firearm being used. The striation marks on bullets are caused by the irregularities in the firearm barrel.

The cartridge case ejected shows marks at the primer (Figure 1) that are caused by the firing pin and the breech face as the cartridge is repelled back in the breach by the force of rifling. The feeding, extraction and ejection mechanisms of the firearm will also leave characteristic marks.

Correspondence : Z.J.M.H. Geradts. Other author information : Email zeno@ holmes.nl; Telephone +31704135681; Fax +31703639238; web http://forensic.to 
In the Forensic Science Institute these marks on cartridge cases and bullets are compared with the test fired ones. Often the cartridge case is the most important forensic specimen in the identification of weapons, as bullets are commonly deformed by the impact. The examiner can also determine, using class characteristics what kind of firearm (often make and model) has been used.
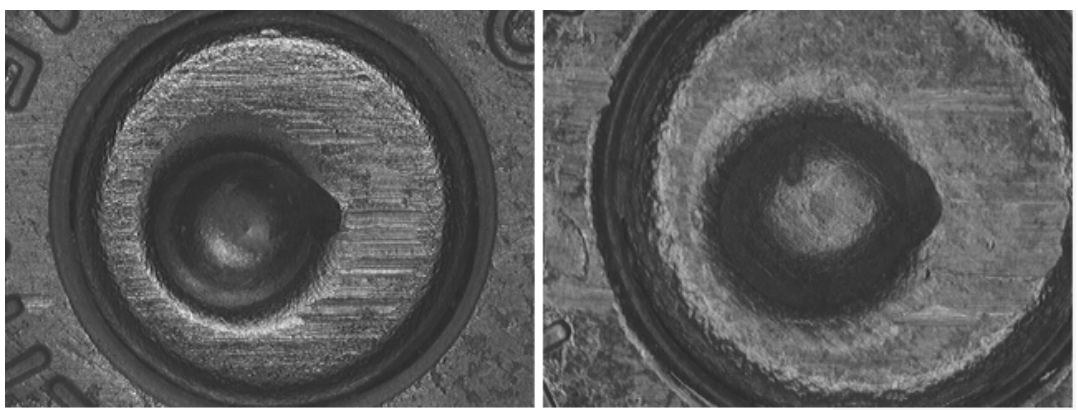

Side light (Breechface)

Ring Light (Firing pin)

Figure 1: Image of breech face with sidelight and image of firing pin with ring light

\section{BALLISTIC IMAGING SYSTEMS}

DRUGFIRE $^{1}$ and IBIS ${ }^{2,3,4,5}$ are databases that can be used for acquiring, storing and analyzing images of bullets and cartridge cases. These two systems have been evaluated at our laboratory.

Both systems capture video images of bullet striations and of the markings left on cartridge cases. These images are used to produce an electronic signature that is stored in a database. The system then compares this signature to that of another fired bullet or cartridge case - or to an entire database of fired bullets and cartridge cases. The user enters the cartridge case in the database for comparison, and can limit the search to using metadata (e.g. caliber, date limit). Then, the system produces a hitlist that shows a ranking of all cartridge cases based on the similarity, as measured by the system, between the cartridge under investigation and the cartridges in the database. The system functions properly if all relevant matches are in the top of the hit list.

The methods of image matching applied in these systems are not known. However patents ${ }^{2,3,4,5}$ applied by one company describe state-of-the-art image matching methods. The system of IBIS is now used most often, and since the images are acquired in a reproducible way by a special kind of lighting, the ring light, it is expected that this system gives the best matching results.

Other systems that have been described on the market are the system Fireball ${ }^{6}$, the French system CIBLE and the Russian system TAIS. These systems also use image-matching techniques.

Three-dimensional measurement of the striation marks by laser triangulation ${ }^{7}$ or by fusing the images with different angles of coincidence are described in the literature ${ }^{8}$. Since the firearm examiner is used to comparing side light images and not three-dimensional images, development and acceptance of 3D-image matching methods progresses slowly. Therefore this studies is focused on the matching of side light and ring light images.

\section{IMAGE MATCHING}

For this research we tested image-matching techniques, which are available from the literature ${ }^{9}$. There has been much interest in searching of image databases. Several commercial and research systems as QBIC, Virage and Photobook exist ${ }^{10,11}$. These systems search in the contents of images. They generally take features from images, and index these features as descriptors that are easily searchable. The results of searching these databases are generally influenced by the following differences between two matching images:

- Noise

- Rotation and shift

- Difference in light source 
Further differences that are typical for databases of cartridge cases:

- Difference in cartridge case metal (material, type, brand)

- Wear of firearm

- Wear of cartridge case

- Marks between two shots can be different for statistical reasons in the shooting process; this means that sometimes parts of striation and impression marks are visible that are not visible with the next shot

In forensic investigations, the firearm examiner determines which marks on cartridge cases are similar. The approach of this research is a combination of shape of the firing pin and the texture of the impression marks. Since the light conditions and the image of the marks do change depending on the marks, methods have been implemented that compare the grayscale images. In practice it turned out to be important to have an appropriate preprocessing image step to compensate for the variation of light. In the optimal situation the algorithm should only compare the marks caused by the firearm, and not any other features of the cartridge case, as damage and symbols.

In this research approaches are implemented that are both pixel based and feature based. The reason to use featurebased methods is to improve the calculating speed and to keep the selection restricted to marks.

\section{TEST DATABASE}

For our evaluation of image matching algorithms we studied two kinds of images (Figure 1):

- Images of breech faces which are illuminated with side light

- Images of firing pins which are illuminated with ring light

We used a database of 4966 images, which were acquired by different firearm examiners from different institutes around the world using the Drugfire system under different circumstances (light sources and several views of the cartridge case). Table 1a shows the different calibers and the kind of images (side or ring light images). We tested the algorithms on all images (without prior knowledge).

The database consists of side light images of 49 different cartridge cases that have a known match. They are consistent in light conditions. These cartridge cases were fired from 18 different firearms of caliber's 9 mm Parabellum (15), .45 automatic (2) and .32 (1). Depending on the case, there are 2-5 similar marks on the cartridge cases. Some of these cartridge cases are from different test shots. The marks of the cartridge cases and the shapes of the firing pin are visually similar between the matches. These cartridge cases can be mixed with the rest of the database for the experiments with large databases. Five cartridge cases have a rotation of more than 10 degrees to each other. The 49 cartridge cases are also available as ring light images of the firing pin. There are marks in all ring light images of the firing pin that can be distinguished from each other visually.
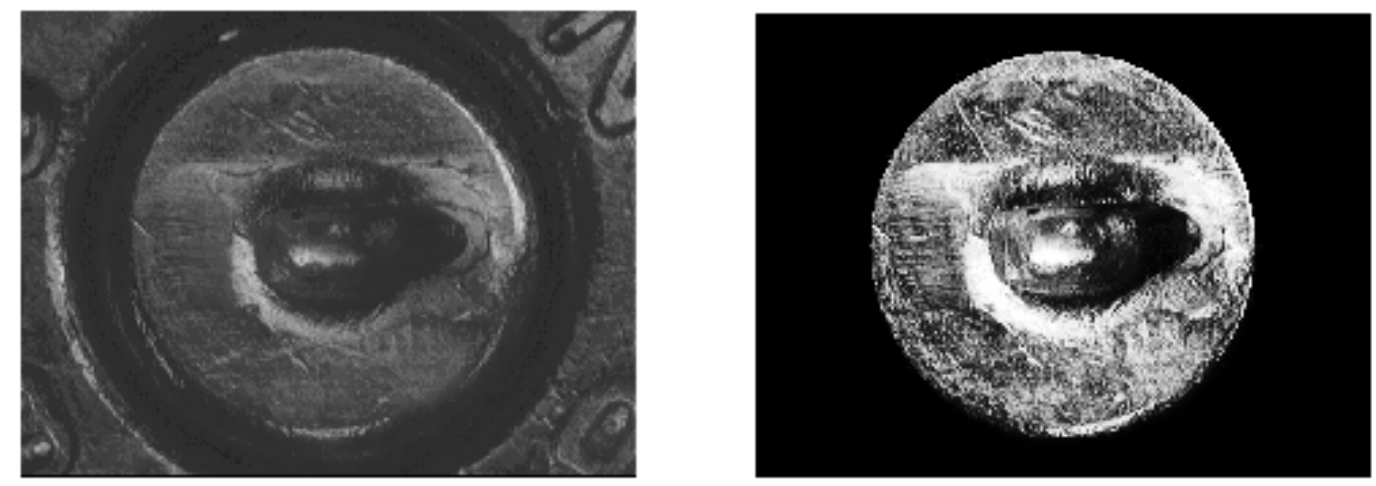

Figure 2: Preprocessing operation (left: original image, right: processed image) 


\section{PREPROCESSING}

\section{Equalization and masking}

We first equalize the images, since the conditions of lighting differed for the cartridge cases in the database. On all images histogram equalization is used in an effort to compensate for differences in lighting conditions.

Since we would like to compare just the inner circle of the image (were most impression marks are), we select the circle manually and all pixels outside of this circle will get a zero gray value (Figure 2). This pre-processing has been carried out to all images that are in our databases.

Because a circular shape is compared, the image can be converted to polar coordinates. The polar image is calculated from the center of the firing pin, and in this way a polar image can be calculated. Since polar coordinates are used, the firing pin will cover a larger area in this image then with regular images (Figure 3 ). For our computation we have selected a 360x360 (angle x radius) image. The polar images are more appropriate for calculation, as the outside of the circle does not have to be modified. The polar images can be selected optionally instead of the regular images.
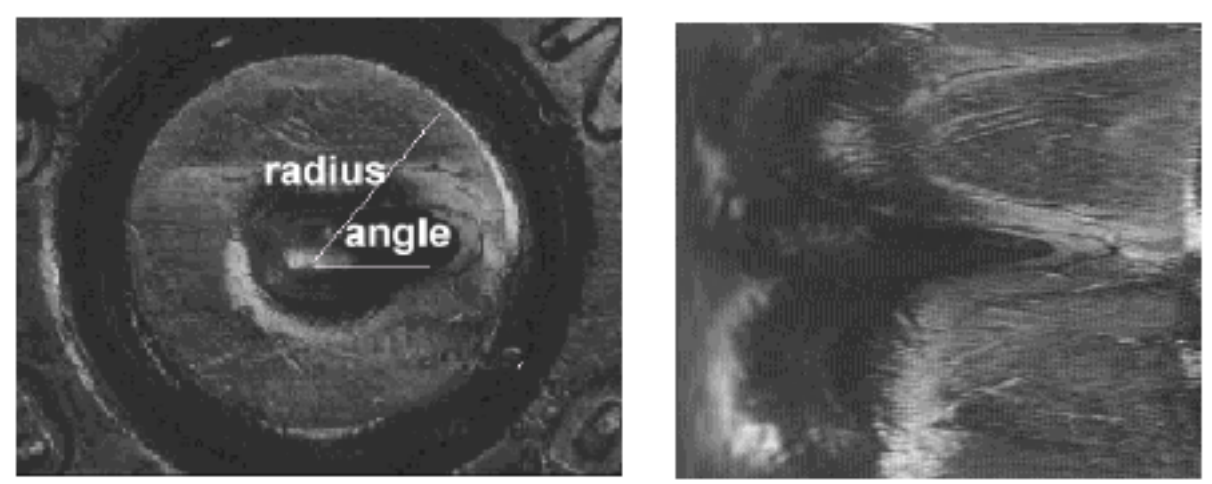

Figure 3: Polar coordinate image of cartridge case $(\mathrm{r}, \Phi)$

\section{Wavelets}

There is a huge number of articles ${ }^{12}$ on pattern recognition based on wavelet transforms. A wavelet transform is a localized function of mean zero. Often wavelets incorporate a pyramidal visualization. Wavelet functions are often wavelike but clipped to a finite domain.

Wavelet transforms are computationally efficient and they allow exact reconstruction of the original data. The reason for choosing a wavelet transform is that we can filter out the information of marks. Since wavelets can work as good filters for different details (from coarse to fine) in the image, the challenge is to choose a wavelet scale and type that is optimal for our marks (fine striation and impression marks).

We have chosen a wavelet transform that works on discrete data that is known as à trous (with holes) algorithm ${ }^{13}$. In this wavelet function the data is sampled by means of a smoothing function, the B3 spline. The different scales of à trous that are calculated can be added to each other and the result will be the original image.

The scale 1 of the à trous algorithm will give the finest details and the noise in the image. The higher scales will give the course variation of lighting in the image. In Figure 4 an example is given of four scales for a cartridge case computed with the à trous algorithm. In the third scale most information of the mark is given. 


\section{IMAGE MATCHING METHODS}

In this section an overview is given of different image matching methods that worked for this database.

\section{Difference of two images}

For a computationally simple kind of comparison we take the variance of the difference in gray values between two images (which is also used in previous research ${ }^{14}$ ).

The hit list is created by sorting the variance from small values for the best matching to high values for images that do not match that well.

Given that the user of the database has to position the cartridge cases with the standard procedure, it might even be positioned 180 degrees rotated. This is caused when the examiner finds the wrong position of the cartridge case in the firearm based on the marks. Also small rotation angles are allowed. We tested the results by rotation of the cartridge case. It appeared that our results are invariant for rotation of up to 5 degrees. The average standard deviation is low and the image that we tested will be retrieved in this situation. The images that are digitized in our laboratory are digitized by using the protocol, however with the other images, it is not completely sure if the protocol is exactly followed, since they are acquired in many different places in the world.

\section{Subtraction}

We subtracted a given image from each image in the database and compared the standard deviation of the result. It appeared that 21 out of the 49 images were in the top positions. 15 were in the top 5 percent of the database. This means that the examiner should browse through 250 cartridge cases before knowing there is no match. This does not work well in practice, since it is a time consuming work. Five cartridge cases were in the top 50 percent of the database. These five cartridge cases had a rotation of more than 10 degrees, and this caused the difference. For this reason this approach is not effective. It appeared that the influence of the shadows and marks in the firing pin could disturb the results of image matching. One of the reasons is that the firing pin mark is a deep mark, and that the shadows and the visual marks in the
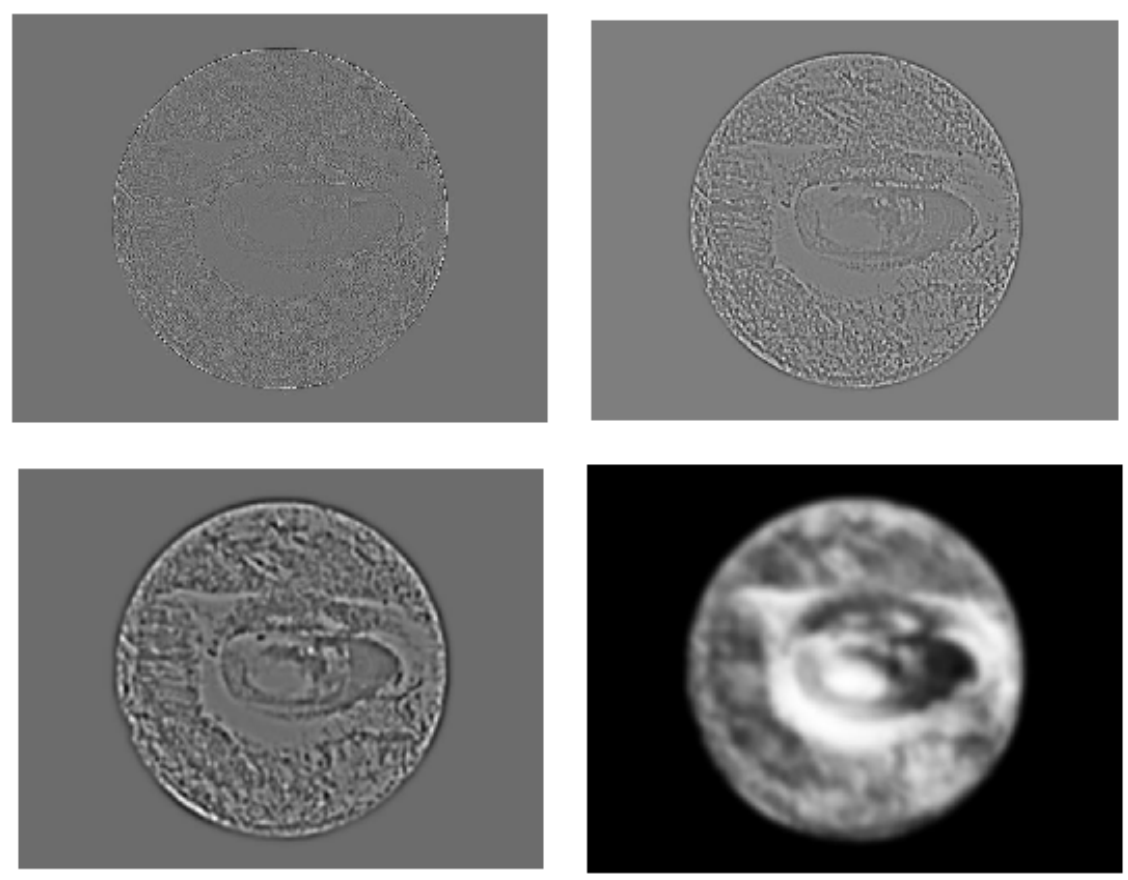

Figure 4: Four scales computed with the à trous wavelet transform 
firing pin are of influence on the final matching result. There is an alternative, namely to filter the firing pin mark out of the image, however the shape of the firing pin is also important for class characteristics. The shape is just a fast way of pre-selection. For the brute force way of comparison it appeared that if we reduce the influence of the correlation of the primer area with fifty percent that the final correlation results improved. We have tested different percentages for these 49, and an optimum was found around fifty percent. If both ring light images exist and side light images, the influence of the firing pin can also be reduced to 0 percent, since the ring light image will show a good image of the shape of the firing pin. The correlation results of the ring light images and of the side light images should be combined.

\section{Compensating for rotation and translation}

From examination of the images, it appeared that some of these images were slightly rotated and translated. For this reason we tried to compensate this influence by rotating and translating the images themselves in the memory of the computer, and calculating the minimum of the standard deviation of the difference in gray values. With those compensations, it appeared that all images were found in the top positions. This approach worked both in the polar coordinates as well as in the raw images. The computation is done by rotating 360 degrees in steps of one degree and shifting 40 pixels in $\mathrm{x}$ and $\mathrm{y}$-direction in steps of one pixel. The computation took more than one month on a Pentium-II PC for 49 images; for this reason this method has not been tested with the complete database.

\section{Log Polar}

A classical technique for registering two images with translation misalignment involved calculating the 2D cross-image matching function ${ }^{15}$. Image registration algorithms are important for this kind of research, since the position of the marks is not known exactly.

The maximum of this function yields the translation necessary to bring the images into alignment. This function has the disadvantage of being sensitive to rotation and scale change. Even small rotations of a few degrees can reduce the peak of the cross image matching function to the noise level.

By using the invariant image descriptors in place of the original images, it is possible to avoid this problem. One such descriptor is the log-polar transform of the Fourier magnitude, which removes the effect of rotation, and uniform scaling into shifts in orthogonal directions ${ }^{16}$.

Results

We tested the log polar transform on raw images. It appeared that 5 out of the 49 cartridge cases were in the top position. All images were however in the first 6 percent (top 300) of the complete database. For this reason the method can be used as a faster selection method. The log polar transform took 7 days to calculate for the complete database of 4190 images.

Better results were found when using the third scale of the à trous transform on these images. All matching images were in the top positions. This method produced similar results as the brute force calculation.

\section{Selecting features by tracking (KLT - method)}

There are extensive studies of image matching for tracking ${ }^{17}$. Since tracking has many similarities with searching in a database of images ${ }^{18}$, we have tested these algorithms. Often these algorithms are optimized for their speed. The features that are of interest are followed. Determining if a feature is of interest can be used for ranking it in a hit list. Tracking also has the problem of registration, since the camera might move, with image intensities that change in a complex way.

One of the methods that appears to work for a wide range of tracking problems, and that works fast, is the Kanade Lucas Tomasi (KLT) equation ${ }^{19}$. Good features (in this approach this means prominent features, as strong edges) are located by examining the minimum eigenvalue of each 2 by 2 -gradient matrix. The features are tracked using a Newton-Raphson method of minimizing the difference between the two windows. Multiresolution tracking allows for even large displacements between images.

Based on this equation, the details in the images that are prominent are selected as points. From each image these points are calculated once and stored in the database. Then the position of those points is compared as a signature. The number of points that are matched between two cartridge cases is a measure of similarity between the two images. 
We can work with this method using the equalized images. Due to noise and variation in the images, the features are not reproducible that well. For this reason, we have combined this with the different scales of images. In each image 100 features are selected and compared to the other images.

It appeared that some images were low in the hit list, because of variations in the light conditions for the raw images, and at scales 1 and 2 it appeared that there were misses. This means that no image matching points were found. The scale 3 appeared to work fine for these marks both for ring light and side light.

\section{CONCLUSIONS AND DISCUSSION}

We tested different image matching algorithms for marks on cartridge cases, using 49 cartridge cases from 18 different firearms. For each match several firearm examiners determined that they were shot with the same firearm.

In cases where the positioning, and the light conditions among the marks in the cartridge cases was reproducible, a simple computation of the standard deviation of the subtracted gray levels put the matching images on top of the hit list. For images that were rotated and shifted, we have built a "brute force" way of image translation and rotation, and the minimum of the standard deviation of the difference is computed. For images that did not have the same light conditions and were rotated relative to each other, it was useful to use the third scale of the "à trous"-multiresolution computation.

From our experiments we conclude that the most optimal method for comparison is possible by a combination of correlation methods. The preprocessed images with the third scale à trous wavelet in combination with the log polar transform worked best. To improve the speed of image matching the KLT-method could be used first for the top five percent for a faster pre-selection. After this log polar correlation can be used, and then it is possible to have a result in a few minutes. Furthermore based on the results of the log polar correlation, a brute force method can be used for the top matching images. The images and image matching methods that are used have marks that can be distinguished visually.

For further improvement, it might be useful to have the refinement in which the user selects the areas that are relevant on the cartridge case for their marks. Sometimes the firearm examiner can have more information that some marks on the cartridge cases are due to damage not caused by the firearm. Examples of this damage are text imprints in the firing pin.

The use of optical processors ${ }^{20,21}$ or parallel processors implemented in hardware is an option to improve the speed of the image matching compared to our brute force method.

\section{ACKNOWLEDGEMENTS}

For this research we would like to thank Andre Hoogstraaten for his contribution in the statistical part. Furthermore we would like to thank the users of the Drugfire database for entering the images in the database for the experiments in this paper.

\section{REFERENCES}

1. B.C. Jones, Intelligent Image Capture of Cartridge Cases for Firearms Examiners, Proceedings SPIE, 2942, pp. 94-104, 1997.

2. R. Baldur, Method for monitoring and adjusting the position of an object under optical observation for imaging, US patent 5,633,717, 1996.

3. R. Baldur, Method and apparatus for obtaining a signature from a fired bullet, US patent 5,659,489, 1997.

4. R. Baldur, Fired cartridge examination method and imaging apparatus. US patent 5,654,801, 1995.

5. R. Baldur, Computer automated bullet analysis apparatus, US patent 5,390,108, 1991.

6. C.L. Smith, Fireball: A Forensic Ballistics Imaging System, Proceedings of the IEEE International Carnahan Conference on Security Technology, 1997, pp 64-70.

7. J. De-Kinder, M. Bonfanti, Automated Comparisons of Bullet Striations Based on 3D Topography, Forensic Science International, 101 (1999), 85-93.

8. F. Puente Leon, Automatische Identifikation von Schußwaffen, Fortschritt-Berichte VDI, Reihe 8, Nr. 787, Düsseldorf, VDI-Verlag, ISBN 3-18-378708-3, 1999.

9. C.L. Smith, Optical imaging techniques for ballistic specimens to identify firearms, Proceedings of IEEE International Carnahan Conference on Security Technology, pp. 275-289, 1995. 
10. Faloutsos C, Barber R, Efficient and Effective Querying bylimage Content, Journal of Intelligent Information Systems, $\mathbf{5}$ pp. 231-262, 1995.

11. M.S. Lew, Content Based Image retrieval : optimal keys, texture, projections and templates, Image Databases and Multimedia Search in Image Databases and Multi-Media Search (Series on Software Engineering and Knowledge Engineering, Vol 8) by Smeulders A, Jain R, ISBN 981-02-3327-2, pp. 39-47, 1999.

12. J.L. Starck, F. Murtagh, A. Bijaoui, Image Processing and Data Analysis, the Multiscale Approach, University Press, Cambridge, ISBN 052159914 8, 1998.

13. M. Holschneider, A real-time algorithm for signal analysis with the help of the wavelet transform, in J.M. Combes e.a., editors. Wavelets: Time Frequency Methods and Phase Scale, Springer Verlag, Berlin, pp. 286-297, 1989.

14. Z. Geradts, J. Keijzer; I. Keereweer; A New Approach to Automatic Comparison of Striation Marks, Journal of Forensic Sciences, 39, pp. 974 -982, 1994.

15. P.E. Anuta, Spatial Registration of multispectral and multitemporal digital imagery using fast Fourier transform techniques, IEEE Trans Geo Elec, 8, pp. 353-368, 1974.

16. D. Casasent, D. Psaltis, Position, rotation and scale invariant optical image matching, Applied Optics 15, pp. 1795-1799, 1976.

17. J. Shi and C. Tomasi, Good Features to Track, Proc. IEEE Conf. Computer Vision and Pattern Recognition, pp. 593600, Seattle, June 1994.

18. S. Bres, J.M. Jolion, Detection of Interest Points for Image Indexation, Proceedings of third international conference VISUAL'99, Amsterdam, , Lecture notes in computer science, 1614, Springer-Verlag, pp. 427434, June 2-4, 1999.

19. S. Birchfield, Derivation of Kanade-Lucas-Tomasi Tracking Equation, Stanford, May 1996, http://vision.stanford.edu/ birch/klt/derivation.ps, 1996.

20. J.P. Karins, S.A. Mills, R.B. Dydyk, Optical processor for fingerprint identification, Proceedings of the SPIE - The International Society for Optical Engineering Conference, Proceedings SPIE, 2940 1997, pp. 108-115, 1997.

21. A. Perez-Poch, J. Velasco, Cheap and reconfigurable PC-board for real-time optical Applications using liquid crystal displays, Proceedings of SPIE, 2774, 757-65, 1997. 